PROCEEDINGS OF THE

AMERICAN MATHEMATICAL SOCIETY

Volume 139, Number 8, August 2011, Pages 3015-3024

S 0002-9939(2011)10726-3

Article electronically published on January 13, 2011

\title{
ON GEODESICS OF FINSLER METRICS VIA NAVIGATION PROBLEM
}

\author{
LIBING HUANG AND XIAOHUAN MO
}

(Communicated by Jianguo Cao)

\begin{abstract}
This paper is devoted to a study of geodesics of Finsler metrics via Zermelo navigation. We give a geometric description of the geodesics of the Finsler metric produced from any Finsler metric and any homothetic field in terms of navigation representation, generalizing a result previously only known in the case of Randers metrics with constant $S$-curvature. As its application, we present explicitly the geodesics of the Funk metric on a strongly convex domain.
\end{abstract}

\section{INTRODUCTION}

A smooth curve in a Finsler manifold is called a geodesic if it is locally the shortest path connecting any two nearby points on this curve (cf. 21]). Recently, R. Bryant [5] showed that a Finsler metric on $S^{2}$ of constant flag curvature $K=1$ with reversible geodesics is actually a Riemannian one. M. Crampin's result tells us that if a Randers metric $\alpha+\beta$ has reversible geodesics, then $\beta$ is closed 8 . C. Robles investigated geodesics of Randers metrics of constant $S$-curvature [17]. Randers metrics of constant flag curvature (or quadratic Riemann curvature) have constant $S$-curvature 3, 10. In terms of the navigation representation, they are produced from Riemannian metrics and homothetic vector fields $[13,16,22,23$. In fact, C. Robles classified geodesics in Randers manifolds of constant flag curvature 17.

The aim of this paper is to give a geometric description of the geodesics of the Finsler metric $\tilde{F}$ obtained from an arbitrary Finsler metric $F$ and an arbitrary homothetic field $V$ of $F$ in terms of the navigation representation. Precisely we show the following:

Theorem 1.1. Let $F=F(x, y)$ be a Finsler metric on a manifold $M$ and let $V$ be a vector field on $M$ with $F\left(x, V_{x}\right)<1$. Suppose that $V$ is homothetic with dilation c. Let $\tilde{F}=\tilde{F}(x, y)$ denote the Finsler metric on $M$ defined in (3.20). Then the geodesics of $\tilde{F}$ are given by $\psi_{t}(\gamma(a(t)))$ where $\psi_{t}$ is the flow of $-V, \gamma(t)$

Received by the editors June 30, 2010 and, in revised form, August 3, 2010.

2010 Mathematics Subject Classification. Primary 58B20.

This work is supported by the National Natural Science Foundation of China 11071005

The second author is the corresponding author.

(C)2011 American Mathematical Society Reverts to public domain 28 years from publication 
is a geodesic of $F$ and $a(t)$ is defined by

$$
a(t):= \begin{cases}\frac{e^{2 c t}-1}{2 c}, & \text { if } c \neq 0 \\ t, & \text { if } c=0 .\end{cases}
$$

Our result generalizes a theorem previously only known in the case of Randers metrics with constant $S$-curvature [17. As its application, we represent explicitly the geodesics of the Funk metric on a strongly convex domain (see Proposition 4.1).

Recall that a vector field $V$ on a Finsler manifold $(M, F)$ is said to be a homothetic field of $F$ with dilation $c$ if the corresponding flow $\phi_{t}$ is homothetic with dilation $c$. In particular $V$ is called a Killing field if $c=0$.

It is worth mentioning our recent result that for a non-Killing homothetic field $V$, the navigation representation has the flag curvature decreasing property [15].

For interesting results of geodesics on Finsler spheres, we refer the reader to [1, 9, 11.

\section{Preliminaries}

Let us recall firstly the definition of the Finsler metrics.

Definition 2.1 ([2]). Let $M$ be a finite-dimensional manifold. A function $F$ : $T M \rightarrow[0,+\infty)$ is a Finsler metric if it satisfies

(a) $F$ is $C^{\infty}$ on $T M \backslash\{0\}$;

(b) $F(x, \lambda y)=\lambda F(x, y)$ for all $y \in T_{x} M, x \in M$ and $\lambda>0$;

(c) for every $y \in T_{x} M \backslash\{0\}$, the quadratic form

$$
\left.g_{x, y}(u, v) \equiv \frac{1}{2} \frac{\partial^{2}}{\partial s \partial t} F^{2}(x, y+s u+t v)\right|_{t=s=0}, \quad \forall u, v \in T_{x} M
$$

is positive definite.

In this case, $(M, F)$ is called a Finsler manifold. Let $S M$ be the projective sphere bundle of $M$, obtained from $T M$ by identifying nonzero vectors which differ from each other by a positive multiplicative factor. Each geometrical quantity on $T M$, homogeneous of degree zero, is considered to sit on $S M$. Define

$$
\omega:=F_{y^{i}} d x^{i} .
$$

Then $\omega$ is a differential form on $S M$. It is easy to verify that

$$
\omega \wedge(d \omega)^{n-1} \neq 0, \quad n=\operatorname{dim} M
$$

(cf. [6]), i.e., that $\omega$ defines a contact structure on $S M$. This form $\omega$ is known in the calculus of variations as the Hilbert form.

Since $\omega$ is a contact form, there exists a unique vector field $X$ on $S M$ that satisfies

$$
\omega(X)=1, \quad X\lrcorner(d \omega)=0 .
$$

This vector field $X$ is known as the Reeb vector field [4]. It is easy to see that a $C^{\infty}$-curve is a (constant Finslerian speed) geodesic if its canonical lift in $S M$ is an integral curve of the Reeb vector field $X$ [4].

Every vector $y \in T_{x} M \backslash\{0\}$ uniquely determines a covector $p \in T_{x}^{*} M \backslash\{0\}$ by

$$
p(u):=\left.\frac{1}{2} \frac{d}{d t}\left(F^{2}(x, y+t u)\right)\right|_{t=0}, \quad u \in T_{x} M .
$$

The resulting map $L_{x}^{F}: y \in T_{x} M \rightarrow p \in T_{x}^{*} M$ is called the Legendre transformation at $x$. The family $L^{F^{*}}:=\left\{L_{x}^{F} \mid x \in M\right\}$ is called the Legendre transformation. 
Define a non-negative scalar function $H=H(x, p)$ by

$$
H(x, p):=\max _{y \in T_{x} M \backslash\{0\}} \frac{p(y)}{F(x, y)} .
$$

The function $H$ is $C^{\infty}$ on $T^{*} M \backslash\{0\}$ and $H_{x}:=\left.H\right|_{T_{x}^{*} M}$ is a Minkowski norm on $T_{x}^{*} M$ for $x \in M$. Such a function is called a Cartan metric [12, 15] (co-Finsler metric in an alternative terminology [18, 19]). The pair $(M, H)$ is called a Cartan manifold.

Every covector $p \in T_{x}^{*} M \backslash\{0\}$ uniquely determines a vector $y \in T_{x} M \backslash\{0\}$ by

$$
q(y):=\left.\frac{1}{2} \frac{d}{d t}\left(H^{2}(x, p+t q)\right)\right|_{t=0}, \quad q \in T_{x}^{*} M .
$$

The resulting map $L_{x}^{F *}: p \in T_{x}^{*} M \rightarrow y \in T_{x} M$ is called the inverse Legendre transformation at $x$. Indeed $L_{x}^{F}$ and $L_{x}^{F *}$ are inverses of each other. Moreover, they preserve the Minkowski norms

$$
H(x, p)=F\left(x,\left(L_{x}^{F}\right)^{-1} p\right) .
$$

Recently, one of the important approaches in discussing the Finsler metric is the (Zermelo) navigation problem. For instance, Bao-Robles-Shen have classified Randers metrics of constant flag curvature via the navigation problem in a Riemannian manifold 3 .

The main technique of the navigation problem is described as follows. Given a Finsler metric $F$ and a vector field $V$ with $F\left(x, V_{x}\right)<1$, define a new Finsler metric $\tilde{F}$ by

$$
F\left(x, \frac{y}{\tilde{F}(x, y)}+V_{x}\right)=1, \quad \forall x \in M, y \in T_{x} M .
$$

A (local) flow (a local one-parameter group in an alternative terminology) on a manifold $M$ is a map $\phi:(-\epsilon, \epsilon) \times M \rightarrow M$, also denoted by $\phi_{t}:=\phi(t, \cdot)$, satisfying

- $\phi_{0}=\mathrm{id}: M \rightarrow M$

- $\phi_{s} \cdot \phi_{t}=\phi_{s+t}$ for any $s, t \in(-\epsilon, \epsilon)$ with $s+t \in(-\epsilon, \epsilon)$.

Hence, the lift of a flow $\phi_{t}$ on $M$ is a flow $\hat{\phi}_{t}$ on $T^{*} M$,

$$
\hat{\phi}_{t}(x, p):=\left(\phi_{t}(x),\left(\phi_{t}^{*}\right)^{-1}(p)\right) .
$$

By the relationship between vector fields and flows, (2.2) induces a natural way to lift a vector field $V$ on $M$ to a vector field $X_{V}^{*}$ on $T^{*} M$.

A vector field $V$ on a Finsler manifold $(M, F)$ is called homothetic with dilation $c$ if its flow $\phi_{t}$ satisfies

$$
F\left(\phi_{t}(x), \phi_{t *}(y)\right)=e^{2 c t} F(x, y), \quad \forall x \in M, y \in T_{x} M .
$$

Similarly, a vector field $V$ on a Cartan manifold $(M, H)$ is called homothetic with dilation $c$ if its flow $\phi_{t}$ satisfies

$$
H\left(\phi_{t}(x),\left(\phi_{t}^{*}\right)^{-1}(p)\right)=e^{-2 c t} H(x, p), \quad \forall x \in M, p \in T_{x}^{*} M .
$$

Lemma 2.2. Let $V$ be a homothetic field on a Finsler manifold $(M, F)$ with dilation $c$ and $H$ its Cartan metric defined by (2.1). Then $V$ is a homothetic field of $H$ with dilation $c$. 
Proof. By using (2.1) and (2.3) we have

$$
\begin{aligned}
H\left(\phi_{t}(x),\left(\phi_{t}^{*}\right)^{-1}(p)\right) & =\max _{\tilde{y} \in T_{\phi_{t}(x)} M \backslash\{0\}} \frac{\left[\left(\phi_{t}^{*}\right)^{-1}(p)\right](\tilde{y})}{F\left(\phi_{t}(x), \tilde{y}\right)} \\
& =\max _{\tilde{y} \in T_{\phi_{t}(x)} M \backslash\{0\}} \frac{p\left(\left(\phi_{t *}\right)^{-1}(\tilde{y})\right)}{F\left(\phi_{t}(x), \tilde{y}\right)} \\
& =\max _{y \in T_{x} M \backslash\{0\}} \frac{p(y)}{F\left(\phi_{t}(x), \phi_{t *}(y)\right)} \\
& =\max _{y \in T_{x} M \backslash\{0\}} \frac{p(y)}{e^{2 c t} F(x, y)} \\
& =e^{-2 c t} \max _{y \in T_{x} M \backslash\{0\}} \frac{p(y)}{F(x, y)}=e^{-2 c t} H(x, p),
\end{aligned}
$$

where $y:=\left(\phi_{t *}\right)^{-1}(\tilde{y})$. It follows that $V$ is a homothetic field of $H$ with dilation $c$.

\section{Geodesics of a Finsler metric via navigation Problem}

In this section, we give a geometric description of geodesics of Finsler metrics via the homothetic navigation problem in a Finsler manifold. First, we show the following:

Lemma 3.1. Let $N$ be a manifold, and let $V$ and $W$ be vector fields on $N$ that satisfy

$$
[V, W]=-c V
$$

for some constant c. Let $\phi_{t}$ and $\psi_{t}$ be local 1-parameter groups of $V$ and $W$ respectively. Then $\psi_{t} \circ \phi_{a(t)}$ is a local 1-parameter group of the vector field $V+W$, where $a(t)$ is defined by

$$
a(t):= \begin{cases}\frac{e^{c t}-1}{c}, & \text { if } c \neq 0 \\ t, & \text { if } c=0 .\end{cases}
$$

Proof. Direct calculations yield

$$
\frac{d a}{d t}=e^{c t} .
$$

Since $V$ is the induced vector field from $\phi$,

$$
\left.\frac{d}{d t} \phi_{t}(x)\right|_{t=t_{0}}=V_{\phi_{t_{0}}(x)} \text {. }
$$

Let $\eta_{t}:=\phi_{a(t)}, u:=a(t)$. By using (3.2) and (3.3) we obtain

$$
\frac{d}{d t}\left[\eta_{t}(x)\right]_{t=s}=\left.\left.\frac{d \phi_{u}}{d u}\right|_{u=a(s)} \frac{d a}{d t}\right|_{t=s}=\left.e^{c s} V\right|_{\phi_{a(s)}(x)}=\left.e^{c s} V\right|_{\eta_{s}(x)} .
$$

From (3.1), one obtains $[W, V]=c V$. It follows that

$$
\begin{aligned}
c \psi_{t *} V & =\psi_{t *}(c V) \\
& =\psi_{t *}[W, V] \\
& =\left[\psi_{t *} W, \psi_{t *} V\right]=\left[W, \psi_{t *} V\right] .
\end{aligned}
$$


This gives

$$
\begin{aligned}
c \psi_{t *} V_{p}(f) & =\left[W, \psi_{t *} V\right]_{p} f \\
& =\left[\left(\mathcal{L}_{W}\left(\psi_{t *} V\right)\right]_{p} f\right. \\
& =\lim _{\Delta t \rightarrow 0} \frac{1}{\Delta t}\left[\psi_{t *} V-\left(\psi_{t+\Delta t}\right)_{*} V\right]_{p} f=-\frac{d}{d t}\left[\psi_{t *} V\right]_{p} f
\end{aligned}
$$

for a point $p \in N$ and a function $f \in \mathcal{C}^{\infty}(N)$. We set

$$
y(t):=\psi_{t *} V_{p}(f) .
$$

Substituting (3.7) into (3.6) yields

$$
\frac{d y}{d t}=-c y
$$

Solving (3.8), we get

$$
y=C_{1} e^{-c t} .
$$

Plugging (3.7) into (3.9) yields

$$
\psi_{t *} V_{p}(f)=C_{1}(p, f) e^{-c t} .
$$

Taking $t=0$, then $C_{1}(p, f)=V_{p} f$. Plugging this into (3.10) yields $\left(\psi_{t}\right)_{*} V_{p}(f)=$ $e^{-c t} V_{p} f$ for any point $p \in N$ and any function $f \in \mathcal{C}^{\infty}(N)$. It follows that

$$
\left(\psi_{t}\right)_{*} V=e^{-c t} V \text {. }
$$

By using (3.4), for any function $f \in \mathcal{C}^{\infty}(N)$ we have

$$
\begin{aligned}
\frac{d}{d t}\left[\psi_{t} \circ \eta_{t}(x)\right]_{t=s} f= & \lim _{\Delta s \rightarrow 0} \frac{1}{\Delta s}\left[f \circ \psi_{s+\Delta s} \circ \eta_{s+\Delta s}(x)-f \circ \psi_{s} \circ \eta_{s}(x)\right] \\
= & \lim _{\Delta s \rightarrow 0} \frac{1}{\Delta s}\left[f \circ \psi_{s+\Delta s} \circ \eta_{s+\Delta s}(x)-f \circ \psi_{s} \circ \eta_{s+\Delta s}(x)\right] \\
& +\lim _{\Delta s \rightarrow 0} \frac{1}{\Delta s}\left[f \circ \psi_{s} \circ \eta_{s+\Delta s}(x)-f \circ \psi_{s} \circ \eta_{s}(x)\right] \\
= & \lim _{\Delta s \rightarrow 0} \frac{f \circ \psi_{s+\Delta s}-f \circ \psi_{s}}{\Delta s}\left(\eta_{s+\Delta s}(x)\right) \\
& +\left(f \circ \psi_{s}\right)_{*}\left(\lim _{\Delta s \rightarrow 0} \frac{\eta_{s+\Delta s}(x)-\eta_{s}(x)}{\Delta s}\right) \\
= & W_{\psi_{s} \circ \eta_{s}(x)} f+\psi_{s *}\left(\left.e^{c s} V\right|_{\eta_{s}(x)}\right) f .
\end{aligned}
$$

It follows that

$$
\frac{d}{d t}\left[\psi_{t} \circ \eta_{t}(x)\right]_{t=s}=W_{\psi_{s} \circ \eta_{s}(x)}+\psi_{s *}\left(\left.e^{c s} V\right|_{\eta_{s}(x)}\right) .
$$

By using (3.11) we have

$$
\psi_{s *}\left(\left.e^{c s} V\right|_{\eta_{s}(x)}\right)=e^{c s} \psi_{s *}\left(\left.V\right|_{\eta_{s}(x)}\right)=e^{c s} e^{-c s} V_{\psi_{s} \circ \eta_{s}(x)}=V_{\psi_{s} \circ \eta_{s}(x)} .
$$

Plugging this into (3.12) yields

$$
\frac{d}{d t}\left[\psi_{t} \circ \eta_{t}(x)\right]_{t=s}=W_{\psi_{s} \circ \eta_{s}(x)}+V_{\psi_{s} \circ \eta_{s}(x)}=(W+V)_{\psi_{s} \circ \eta_{s}(x)} .
$$

It follows that $\psi_{t} \circ \eta_{t}$ is a local 1-parameter group of the vector field $V+W$. 
Lemma 3.2. For a homothetic field $V$ on a Cartan manifold $(M, H)$ with dilation $c$, we have

$$
\left[X^{b}, X_{V}^{*}\right]=2 c X^{b},
$$

where $X^{b}=\left(L^{F}\right)_{*} X$ and $X_{V}^{*}$ is the lift of $V$ to $T^{*} M$.

Proof. In natural coordinates, we have

$$
X_{V}^{*}=v^{i} \frac{\partial}{\partial x^{i}}-p_{j} \frac{\partial v^{j}}{\partial x^{i}} \frac{\partial}{\partial p_{i}},
$$

where $V=v^{i} \frac{\partial}{\partial x^{i}}$ [15, (5.3)]. Note that $V$ is homothetic with respect to $H$ with dilation $c$. Differentiating (2.4) with respect to $t$ at $t=0$ yields

$$
X_{V}^{*}(H)=-2 c H .
$$

By using (3.14), we have

$$
X_{V}^{*}(H)=v^{i} \frac{\partial H}{\partial x^{i}}-p_{j} \frac{\partial v^{j}}{\partial x^{i}} \frac{\partial H}{\partial p_{i}} .
$$

It follows that

$$
v^{i} \frac{\partial H}{\partial x^{i}}-p_{j} \frac{\partial v^{j}}{\partial x^{i}} \frac{\partial H}{\partial p_{i}}=-2 c H .
$$

Differentiating (3.16) with respect to $p_{k}$ gives

$$
v^{i} \frac{\partial^{2} H}{\partial x^{i} \partial p_{k}}-\sum_{i} \frac{\partial v^{k}}{\partial x^{i}} \frac{\partial H}{\partial p_{i}}-\sum_{i} p_{j} \frac{\partial v^{j}}{\partial x^{i}} \frac{\partial^{2} H}{\partial p_{i} \partial p_{k}}=-2 c \frac{\partial H}{\partial p_{k}} .
$$

Differentiating (3.16) with respect to $x^{k}$ yields

$$
\frac{\partial v^{i}}{\partial x^{k}} \frac{\partial H}{\partial x^{i}}+v^{i} \frac{\partial^{2} H}{\partial x^{i} \partial x^{k}}-p_{j} \frac{\partial^{2} v^{j}}{\partial x^{i} \partial x^{k}} \frac{\partial H}{\partial p_{i}}-p_{j} \frac{\partial v^{j}}{\partial x^{i}} \frac{\partial^{2} H}{\partial p_{i} x^{k} \partial=}-2 c \frac{\partial H}{\partial x^{k}} .
$$

By Lemma 4.5 in [15], $X^{b}$ is the Hamiltonian vector field for $H$. Hence it has the local expression

$$
X^{b}=\frac{\partial H}{\partial p_{i}} \frac{\partial}{\partial x^{i}}-\frac{\partial H}{\partial x^{i}} \frac{\partial}{\partial p_{i}} .
$$

It follows from (3.14) and (3.19) that

$$
\begin{aligned}
{\left[X^{b}, X_{V}^{*}\right]=} & {\left[\frac{\partial H}{\partial p_{i}} \frac{\partial}{\partial x^{i}}-\frac{\partial H}{\partial x^{i}} \frac{\partial}{\partial p_{i}}, v^{j} \frac{\partial}{\partial x^{j}}-p_{k} \frac{\partial v^{k}}{\partial x^{j}} \frac{\partial}{\partial p_{j}}\right] } \\
= & {\left[\frac{\partial H}{\partial p_{i}} \frac{\partial}{\partial x^{i}}, v^{j} \frac{\partial}{\partial x^{j}}\right]-\left[\frac{\partial H}{\partial x^{i}} \frac{\partial}{\partial p_{i}}, v^{j} \frac{\partial}{\partial x^{j}}\right] } \\
& -\left[\frac{\partial H}{\partial p_{i}} \frac{\partial}{\partial x^{i}}, p_{k} \frac{\partial v^{k}}{\partial x^{j}} \frac{\partial}{\partial p_{j}}\right]+\left[\frac{\partial H}{\partial x^{i}} \frac{\partial}{\partial p_{i}}, p_{k} \frac{\partial v^{k}}{\partial x^{j}} \frac{\partial}{\partial p_{j}}\right] .
\end{aligned}
$$

Recall that

$$
[f X, g Y]=f(X g) Y-g(Y f) X+f g[X, Y]
$$


for any vector fields $X, Y$ and functions $f, g$. It follows that

$$
\begin{aligned}
{\left[X^{b}, X_{V}^{*}\right]=} & \frac{\partial H}{\partial p_{i}} \frac{\partial v^{j}}{\partial x^{i}} \frac{\partial}{\partial x^{j}}-v^{j} \frac{\partial^{2} H}{\partial p_{i} \partial x_{j}} \frac{\partial}{\partial x^{i}}+v^{j} \frac{\partial^{2} H}{\partial x^{i} \partial x^{j}} \frac{\partial}{\partial p_{i}} \\
& -p_{k} \frac{\partial H}{\partial p_{i}} \frac{\partial^{2} v^{k}}{\partial x^{i} \partial x^{j}} \frac{\partial}{\partial p_{j}}+p_{k} \frac{\partial v^{k}}{\partial x^{j}} \frac{\partial^{2} H}{\partial p_{i} \partial p_{j}} \frac{\partial}{\partial x^{i}} \\
& +\frac{\partial H}{\partial x_{i}} \frac{\partial v^{i}}{\partial x^{j}} \frac{\partial}{\partial p_{j}}-p_{k} \frac{\partial v^{k}}{\partial x^{j}} \frac{\partial^{2} H}{\partial x^{i} \partial p_{j}} \frac{\partial}{\partial p_{i}} \\
= & -\left(v^{j} \frac{\partial^{2} H}{\partial x^{j} \partial p_{k}}-\sum_{i} \frac{\partial v^{k}}{\partial x^{i}} \frac{\partial H}{\partial p_{i}}-\sum_{j} p_{i} \frac{\partial v^{i}}{\partial x^{j}} \frac{\partial^{2} H}{\partial p_{j} \partial p_{k}}\right) \frac{\partial}{\partial x^{k}} \\
& +\left(\frac{\partial v^{i}}{\partial x^{k}} \frac{\partial H}{\partial x^{i}}+v^{j} \frac{\partial^{2} H}{\partial x^{j} \partial x^{k}}-p_{j} \frac{\partial^{2} v^{j}}{\partial x^{i} \partial x^{k}} \frac{\partial H}{\partial p_{i}}-p_{i} \frac{\partial v^{i}}{\partial x^{j}} \frac{\partial^{2} H}{\partial p_{j} \partial x^{k}}\right) \frac{\partial}{\partial p_{k}} \\
= & 2 c \frac{\partial H}{\partial p_{k}} \frac{\partial}{\partial x^{k}}-2 c \frac{\partial H}{\partial x^{k}} \frac{\partial}{\partial p_{k}}=2 c X^{b} .
\end{aligned}
$$

Here we have used (3.17), (3.18) and (3.19).

Proof of Theorem 1.1. Let $V$ be a homothetic field on a Finsler manifold $(M, F)$ with dilation $c$ and let $X$ be the Reeb vector field. Applying the Legendre transformation, we obtain a Cartan metric $H(x, p)$. From Lemma 2.2, $V$ is a homothetic field of $(M, H)$ with dilation $c$. By Lemma 3.2 and (3.14), we have $\left[X^{b}, X_{-V}^{*}\right]=-2 c X^{b}$. Let $\phi_{t}$ and $\hat{\psi}_{t}$ be local 1-parameter groups of $X^{b}$ and $X_{-V}^{*}$ respectively. By using (3.14) we get $X^{b}+X_{-V}^{*}=X^{b}-X_{V}^{*}$. Taking this together with Lemma 3.1, we obtain that $\hat{\psi}_{t} \circ \phi_{a(t)}$ is a local 1-parameter group of $X^{b}-X_{V}^{*}$, where

$$
a(t)= \begin{cases}\frac{e^{2 c t}-1}{2 c}, & \text { if } c \neq 0 \\ t, & \text { if } c=0 .\end{cases}
$$

Recall that a navigation problem makes use of a Finsler metric $F$ and a vector field $V$ with $F\left(x, V_{x}\right)<1$ and produces a new Finsler metric $\tilde{F}$ by solving the equation

$$
F(x, y+\tilde{F}(x, y) V)=\tilde{F}(x, y) .
$$

Let $\tilde{X}$ be the Reeb vector field of $\tilde{F}$ and $\tilde{X}^{b}=\left(L^{\tilde{F}}\right)_{*} \tilde{X}$. By Lemma 6.2 in [15, we have

$$
\tilde{X}^{b}=X^{b}-X_{V}^{*}
$$

It follows that $\hat{\psi}_{t} \circ \phi_{a(t)}$ is a local 1-parameter group of $\tilde{X}^{b}$.

Since $L^{F}(x, y)=\left(x, L_{x}^{F}(y)\right)$, we see that any geodesic of $F$ is precisely the projection of an integral curve of $X^{b}$. Similarly, a geodesic of $\tilde{F}$ is precisely the projection of an integral curve of $\tilde{X}^{b}$. Note that

$$
\hat{\psi}_{t}(x, p)=\left(\psi_{t}(x),\left(\psi_{t}^{*}\right)^{-1}(p)\right),
$$

where $\psi_{t}$ is the flow produced by $-V$.

Let $\pi: T^{*} M \backslash\{0\} \rightarrow M$ be the natural projection. It follows that

$$
\pi \circ \hat{\psi}_{t}(x, p)=\pi\left(\psi_{t}(x),\left(\psi_{t}^{*}\right)^{-1}(p)\right)=\psi_{t}(x)=\psi_{t} \circ \pi(x, p)
$$


for any $x \in M$ and $p \in T_{x} M \backslash\{0\}$. Hence we have

$$
\pi \circ \hat{\psi}_{t}=\psi_{t} \circ \pi \text {. }
$$

It follows that

$$
\pi \circ \hat{\psi}_{t} \circ \phi_{a(t)}=\psi_{t} \circ \pi \circ \phi_{a(t)}=\psi_{t}(\gamma(a(t))),
$$

where $\gamma(t):=\pi\left(\phi_{t}(x)\right)$. Thus we have proved Theorem 1.1.

Remark 3.1. The reader should note that the navigation problem adopted here differs from those of C. Robles and Z. Shen [17, 20], where the navigation problem is defined by

$$
F\left(x, \frac{y}{\tilde{F}(x, y)}-V\right)=1 ;
$$

i.e., the $\tilde{F}$ we define with $(F, V)$ is precisely the $\tilde{F}$ that Shen defines with $(F,-V)$.

\section{Geodesics of Funk metrics on Convex domains}

In this section we are going to represent explicitly the geodesics of the Funk metric on a strongly convex domain.

Given a Minkowski norm $\varphi: \mathbb{E} \rightarrow \mathbb{R}$ on a vector space $\mathbb{E}$, one can construct $\Omega:=\{v \in \mathbb{E} \mid \varphi(v)<1\}, T_{x} \Omega \simeq \mathbb{E}$. A domain $\Omega$ in $\mathbb{E}$ defined by a Minkowski norm $\varphi$ is called a strongly convex domain 19 . Thus $(\Omega, F(x, y))$ is a Minkowski manifold, where $F(x, y):=\varphi(y)$. For each $x \in \Omega$, identify $T_{x} \Omega$ with $\mathbb{E}$. Thus $V_{x}:=x$ is a radical vector field on $\Omega$ satisfying $F\left(x, V_{x}\right)=\varphi(x)<1$. Moreover $V$ is a homothetic field of $F$ with dilation $c=\frac{1}{2}$ [15, Example 1]. Define a 1-parameter transformation $\psi_{t}$ on $\Omega$ by

$$
\psi_{t}(x)=e^{-t} x .
$$

Note that $T_{\psi_{t}(x)} \Omega \simeq \mathbb{E}$ for any $t$. It is easy to see that

$$
\psi_{t *}(y)=e^{-t} y, \quad \text { for } \quad \forall y \in T_{x} \Omega .
$$

Thus we have

$$
\begin{aligned}
F\left(\psi_{t}(x), \psi_{t *}(y)\right) & =\varphi\left(\psi_{t *}(y)\right) \\
& =\varphi\left(e^{-t} y\right) \\
& =e^{-t} \varphi(y)=e^{-t} F(x, y)
\end{aligned}
$$

for any $(x, y) \in T \Omega$. It follows that $\varphi_{t}$ is homothetic. A direct calculation yields

$$
\left.\frac{d \psi_{t}(x)}{d t}\right|_{t=0}=-x=-V_{x} .
$$

Thus $\psi_{t}$ is the flow of the vector field $-V$. By using the Minkowski metric $F$ and the homothetic field $V$, we produce a new Finsler metric $\tilde{F}$ in terms of the navigation problem. $\tilde{F}$ is called the Funk metric on a strongly convex domain $\Omega$ (cf. [7, Example 3.4.3]).

Since $F$ is Minkowskian, it is locally projectively flat. This implies that the unit speed geodesic through $x$ with tangent vector $y(\neq 0)$ is $\gamma(t):=x+\frac{t}{\varphi(y)} y$. Note that $V$ is a homothetic field with dilation $c=\frac{1}{2}$. We have

$$
a(t)=e^{t}-1
$$


(see Theorem 1.1). It follows that

$$
\psi_{t}(\gamma(a(t)))=e^{-t}\left[x+\frac{e^{t}-1}{\varphi(y)} y\right] .
$$

By Theorem 1.1, we have the following.

Proposition 4.1. Let $\varphi: \mathbb{E} \rightarrow \mathbb{R}$ be a Minkowski norm and $\Omega$ its strongly convex domain. Assume that $\tilde{F}$ is the Funk metric on $\Omega$ defined by

$$
\varphi\left(\frac{y}{\tilde{F}(x, y)}+x\right)=1 .
$$

Then the geodesics of $\tilde{F}$ are given by

$$
e^{-t}\left[x+\frac{e^{t}-1}{\varphi(y)} y\right]
$$

\section{REFERENCES}

1. V. Bangert and Y. Long, The existence of two closed geodesics on every Finsler 2-sphere, Math. Ann. 346 (2010), 335-366. MR2563691

2. D. Bao, S.-S. Chern and Z. Shen, An introduction to Riemann-Finsler geometry. Graduate Texts in Mathematics, 200. Springer-Verlag, New York, 2000. MR1747675 (2001g:53130)

3. D. Bao, C. Robles and Z. Shen, Zermelo navigation on Riemannian manifolds. J. Differential Geom. 66 (2004), 377-435. MR2106471 (2005k:58023)

4. R. Bryant, Some remarks on Finsler manifolds with constant flag curvature, special issue for S. S. Chern. Houston J. Math. 28 (2002), 221-262. MR.1898190(2003h:53102)

5. R. Bryant, Geodesically reversible Finsler 2-spheres of constant curvature, inspired by S. S. Chern, 95-111, Nankai Tracts Math., 11, World Sci. Publ., Hackensack, NJ, 2006. MR2313331 (2008a:53075)

6. S.-S. Chern, Riemannian geometry as a special case of Finsler geometry, Contemp. Math., 196. Amer. Math. Soc., 1996, 51-58. MR1403576 (98e:53026)

7. S.-S. Chern and Z. Shen, Riemann-Finsler geometry. Nankai Tracts in Mathematics, 6. World Scientific Publishing Co. Pte. Ltd., Hackensack, NJ, 2005. MR2169595 (2006d:53094)

8. M. Crampin, Randers spaces with reversible geodesics, Publ. Math. Debrecen 67 (2005), 401409. MR2162131 (2006e:53130)

9. H. Duan and Y. Long, Multiplicity and stability of closed geodesics on bumpy Finsler 3-spheres, Calc. Var. Partial Differential Equations 31 (2008), 483-496. MR 2372902 (2008m:58025)

10. B. Li and Z. Shen, On Randers metrics of quadratic Riemann curvature, Internat. J. Math. 20 (2009), 369-376. MR2500075 (2010a:53161)

11. Y. Long and H. Duan, Multiple closed geodesics on 3-spheres, Adv. Math. 221 (2009), 17571803. MR 2522828

12. R. Miron, D. Hrimiuc, H. Shimada and S. Sabau, The geometry of Hamilton and Lagrange spaces. Fundamental Theories of Physics, 118. Kluwer Academic Publishers Group, Dordrecht, 2001. MR 1839409 (2002e:53111)

13. X. Mo, A global classification result for Randers metrics of scalar curvature on closed manifolds, Nonlinear Anal. 69 (2008), 2996-3004. MR2452109 (2009k:53195)

14. X. Mo, On some Finsler metrics of constant (or scalar) flag curvature, accepted for publication in Houston Journal of Mathematics.

15. X. Mo and L. Huang, On curvature decreasing property of a class of navigation problems, Publ. Math. Debrecen 71 (2007), 141-163. MR2340039 (2008g:53092)

16. X. Mo and C. Yu, On the Ricci curvature of a Randers metric of isotropic S-curvature, Acta Math. Sin. (Engl. Ser.) 24 (2008), 911-916. MR2428109 (2009b:53128)

17. C. Robles, Geodesics in Randers spaces of constant curvature, Trans. Amer. Math. Soc. 359 (2007), 1633-1651. MR2272144(2007h:53119)

18. Z. Shen, Two-dimensional Finsler metrics with constant flag curvature, Manuscripta Math. 109 (2002), 349-366. MR.1948019 (2003k:53091) 
19. Z. Shen, Landsberg curvature, S-curvature and Riemann curvature. A sampler of RiemannFinsler geometry, 303-355, Math. Sci. Res. Inst. Publ. 50. Cambridge Univ. Press, Cambridge, 2004. MR2132662 (2005k:53132)

20. Z. Shen, Finsler metrics with $\mathbf{K}=0$ and $\mathbf{S}=0$, Canad. J. Math. 55 (2003), 112-132. MR.1952328 (2004e:53112)

21. Z. Shen, Lectures on Finsler geometry, World Scientific Publishing Co., Singapore, 2001. MR:1845637 (2002f:53032)

22. Z. Shen, and H. Xing, On Randers metrics with isotropic S-curvature, Acta Math. Sin. (Engl. Ser.) 24 (2008), 789-796. MR2403114 (2010c:53109)

23. E. Zermelo, Über das Navigationsproblem bei ruhender oder veränderlicher Windverteilung, Z. Angew. Math. Mech. 11 (1931), 114-124.

School of Mathematical Sciences, Nankai University, Tianjin 300071, People's RePUBLIC OF CHINA

E-mail address: huanglb@nankai.edu.cn

Key Laboratory of Pure and Applied Mathematics, School of Mathematical Sciences, Peking University, Beijing 100871, People's Republic of China

E-mail address: moxh@pku.edu.cn 